\title{
SCHWERPUNKTTHEMA: REFERENZKULTUR
}

\section{Kreative Referenzkultur und Urheberrecht im globalen Wandel1}

\author{
Prof. Dr. Nadine Klass, LL.M. (Wellington)*
}

\section{Einführung in die Problematik}

Techniken der Bezugnahme und der Adaption sind schon immer Grundlage des Kulturverständnisses, ${ }^{2}$ ebenso wie referenzielle und transformative Schaffenspraktiken schon immer ein untrennbarer Bestandteil der Kunst- und Kulturgeschichte ${ }^{3}$ waren. Dennoch ist in der heutigen digitalisierten Gesellschaft eine deutliche Zunahme fremdreferenzieller Werke zu verzeichnen. ${ }^{4}$

Ein maßgeblicher Grund hierfür liegt unter anderem in der einfachen Verfügbarkeit sowie Beherrschbarkeit künstlerischer Bearbeitungstechniken im digitalen Raum: ${ }^{5}$ Das Web 2.0 ermöglicht es jedermann, Inhalte und damit auch urheberrechtlich geschützte Werke

1 Einleitung zum Symposium „Kreative Referenzkultur und Urheberrecht im globalen Wandel“ des SFB „Medien der Kooperation“ (Teilprojekt B07 „Medienpraktiken und Urheberrecht - Soziale und juristische Rahmenbedingungen kooperativen und derivativen Werkschaffens im Netz"), gefördert durch die DFG.

* Inhaberin des Lehrstuhls für Bürgerliches Recht, Recht des Geistigen Eigentums sowie deutsches und europäisches Verfahrensrecht an der Universität Mannheim. Besonderer Dank gilt Frau Kamila Kempfert und Herrn Yavor Stamenov für die Unterstützung bei der Erstellung dieses Beitrages.

2 Von Gehlen, Mashup. Lob der Kopie, 2012, S. 19.

3 Döhl/Wöhrer, Einleitung in: Döhl/Wöhrer (Hrsg.), Zitieren, appropriieren, sampeln. Referenzielle Verfahren in den Gegenwartskünsten, 2014, S. 7; vgl. auch die Beispiele bei Klass, ZUM 2016, 801, 802; Dreier, GRUR 2011, 1059, 1060; Döhl, Mashup in der Musik, 2016, S. 11 (zur Abhängigkeit der Musik); von Gehlen, Mashup. Lob der Kopie, 2012, S. 19, 201 (zur Intertextualität); Jenkins, Convergence Culture: Where Old and New Media Collide, 2006, S. 135 (zur Abhängigkeit der amerikanischen Kunst von verschiedenen Volkstraditionen); Schulze in: Dreier/Schulze (Hrsg.), UrhG, 2018, § 24 UrhG Rn. 1.; siehe auch die Beiträge von Lethem, The ecstasy of influence. A plagiarism, Harper's Magazine, 02/2017, abrufbar unter: https://harpers.org/archive/2007/02/the-ecstasy-o f-influence/ (zuletzt abgerufen am 1.3.2019); sowie Ferguson, Everything is a Remix, abrufbar unter: https://www.everythingisaremix.info/watch-the-series (zuletzt abgerufen am 1.3.2019).

4 Vgl. Schulze in: Dreier/Schulze (Hrsg.), UrhG, 2018, § 24 UrhG Rn. 25; hierzu auch Klass, Die Referenz als Teil der Kunstform: populärkulturelle Praktiken und das Urheberrecht in: FS Gernot Schulze, 2017, S. 147; Hoffmann/Klass, Editorial: The Reference as Part of the Art Form. A Turning Point in Copyright Law?, Media in Action 02/2017, S. 32; Summerer, Illegale Fans, 2015, S. 26; Klass, ZUM 2016, 801; Pötzlberger, GRUR 2018, 675; Kempfert/Reißmann, Transformative Works and German Copyright Law as Matter of Boundary Work, Media in Action 02/2017, S. 65.

5 So auch Peifer, Appropriation Art und Fan Art - geknebelte Kreativität oder klare Urheberrechtsverletzung? in: FS Wandtke, 2013, S. 99, 101. 
oder Werkteile zu ändern, neu zu arrangieren oder zu kombinieren und auf diese Weise abhängig kreativ tätig zu werden. Fotos, Videos, Ausschnitte aus Bildern und Filmen, Tonfolgen und Rhythmussequenzen werden auf diese Art und Weise Teil eines spezifischen künstlerischen Transformationsprozesses ${ }^{6}$ der eine Vielzahl abhängiger Schöpfungen hervorbringt.

Die moderne Referenzkultur konnte so zu einem digitalen Massenphänomen avancieren $^{7}$ und einen Raum schaffen, der sich durch partizipative ${ }^{8}$ und nutzergeprägte Kulturformen auszeichnet. ${ }^{9}$ Internet-Memes, ${ }^{10}$ Mashups, Samplings, Fan-Fiction, Appropriation Art, Remixe und Parodien prägen den digitalen öffentlichen Diskurs und können durch HostingPlattformen wie YouTube und Soundcloud oder auf sozialen Netzwerken einfach, schnell und ungehindert verbreitet und durch andere Nutzer ihrerseits neu bearbeitet werden.

Aber nicht nur die geänderten Schaffensrealitäten haben eine Zunahme der ,kreativen Referenzkultur"11 bewirkt. Die gesteigerte Relevanz fremdreferenzieller Werke kann nicht zuletzt auch mit einer sich verändernden Kunstlandschaft begründet werden, in der postmoderne Kunstformen das ästhetische Spiel mit dem Vorgefundenen suchen ${ }^{12}$ und auch die Kunsttheorie zunehmend die Rolle des Originären und Einzigartigen hinterfragt. ${ }^{13}$

6 Klass, Die Referenz als Teil der Kunstform: populärkulturelle Praktiken und das Urheberrecht in: FS Gernot Schulze, 2017, S. 148.

7 Dobusch, Generation Remix: Popkultur und Kunst im rechtsfreien Raum? in: Djordjevic/Dobusch (Hrsg.), Generation Remix: zwischen Popkultur und Kunst, 2014, S. 9.

8 S. hierzu Jenkins/Ito/Boyd, Participatory Culture in a Networked Era: A Conversation on Youth, Learning, Commerce, and Politics, 2016.

9 Vgl. die Begrifflichkeiten bei Wilke, Kombiniere! Variiere! Transformiere! Mashups als performative Diskursobjekte in populären Medienkulturen in: Mundemenke/Ramos Arenas/Wilke (Hrsg.), Mashups: Neue Praktiken und Ästhetiken in populären Medienkulturen, 2015, S. 15.

10 S. hierzu auch Shifman, Meme: Kunst, Kultur und Politik im digitalen Zeitalter, 2014; Ullrich, Das Wetteifern der Bilder: Eine Archäologie der Mem-Kultur, abrufbar unter https://irights.info/artikel/ das-wetteifern-der-bilder-eine-archaeologie-der-mem-kultur/27306 (zuletzt abgerufen am 1.3.2019).

11 von Gehlen, Mashup. Lob der Kopie, 2012, S. 19, beschreibt diese als „Technik der Bezugnahme, des Zitats und der Adaption“; vgl. auch Klass, Die Referenz als Teil der Kunstform: populärkulturelle Praktiken und das Urheberrecht in: FS Gernot Schulze, 2017, S. 147; Hoffmann/Klass, Editorial: The Reference as Part of the Art Form. A Turning Point in Copyright Law?, Media in Action 02/2017, S. 32 .

12 S. auch McGovern, Referenz und Appropriation in der künstlerischen Ausstellungspraxis in: Döhl/ Wöhrer (Hrsg.), Zitieren, Appropriieren, Sampeln. Referenzielle Verfahren in den Gegenwartskünsten, 2014, S. 113: „Nicht die Produktion von einzelnen, originären Kunstobjekten steht (...) im Fokus künstlerischer Verfahren, sondern vielmehr die Arbeit mit dem vorgefundenen Material (...)“; Klass, Die Referenz als Teil der Kunstform: populärkulturelle Praktiken und das Urheberrecht in: FS Gernot Schulze, 2017, S. 148.

13 Podszun, ZUM 2016, 606, 607 f.; Klass, ZUM 2016, 801, 803; S. auch Ullrich, Raffinierte Kunst: Übung vor Reproduktionen, 2009, S. 17, der betont: „Es wäre schon viel erreicht, wenn man im Original künftig nicht mehr nur das Unmittelbare und Ursprüngliche suchte, sondern darin zugleich das Anfängliche, noch Unfertige und Unvollkommene sähe“. 
Festgehalten werden kann jedenfalls, dass aktuell eine Vielzahl an kreativen Prozessen in den unterschiedlichsten Kunst-und Kulturbereichen zu beobachten ist, in denen Neues entsteht, weil auf Altes Bezug genommen wird.

Gleichfalls ist zu konstatieren, dass die Intensität der Bezugnahme nicht nur in quantitativer, sondern auch in qualitativer Hinsicht eine deutliche Steigerung erfahren hat. ${ }^{14}$

\section{Fremdreferenzialität und das Urheberrecht}

Die zunehmende Fremdreferenzialität wird jedoch nicht nur positiv bewertet - vielmehr müssen sich postmoderne Kunstformen sowie Kulturphänomene, die auf dem Werkschaffen anderer aufbauen, nicht selten den Vorwurf der Illegalität, zumindest aber der moralischen Verwerflichkeit ${ }^{15}$ gefallen lassen. Hinzu kommt, dass sie anerkannte (urheberrechtliche) Vorstellungen und Konzepte von Originalität, Werkcharakter, Neuheit sowie Autorschaft in Frage stellen und damit eine ganze Reihe urheberrechtlicher Fragen aufwerfen. ${ }^{16}$

Auch wenn sich ein Großteil dieser partizipativen Schaffenskultur im nicht-kommerziellen, ${ }^{17}$ vereinzelt auch im halb-öffentlichen Bereich abspielt - was dazu führt, dass sie oftmals von jenen, deren Rechte möglicherweise berührt sind, toleriert werden -, bewegt sich das Wirken doch in einem „Graubereich“ und beschert nicht nur den Künstlern, sondern auch den Inhabern der Rechte an vorbestehenden Werken reichlich Unsicherheit.

$\mathrm{Zu}$ klären ist daher die Frage nach einem angemessenen Ausgleich zwischen den Interessen der Originalurheber und denen der nachschaffenden Schöpfer, denn bei der Benutzung, Neuarrangierung, Rekontextualisierung, Interpretation und Kritik vorbestehender urheberrechtlich geschützter Werke oder Werkteile ${ }^{18}$ treffen unterschiedlichste Interessen auf-

14 Insoweit wird auch von dem Phänomen der „Referenzkultur“ gesprochen; vgl. Summerer, Illegale Fans, 2015, S. 26 m.w.N.

15 Siehe beispielsweise die Diskussion um Richard Prince: http://www.spiegel.de/kultur/gesellschaft/ der-kuenstler-richard-prince-verkauft-fremde-instagram-fotos-a-1035383.html: „Kreative Kunst oder dreister Diebstahl?“ (zuletzt abgerufen am 12.4.2019); zudem: Klass, Die Referenz als Teil der Kunstform: populärkulturelle Praktiken und das Urheberrecht in: FS Gernot Schulze, 2017, S. 148; dies., ZUM 2016, 801, 802 m.w.N.

16 Ähnlich auch McGovern, Referenz und Appropriation in der künstlerischen Ausstellungspraxis in: Döhl/Wöhrer (Hrsg.), Zitieren, appropriieren, sampeln. Referenzielle Verfahren in den Gegenwartskünsten, 2014, S. 116; Großmann, Reproduktionsmusik und Remix-Culture in: Saxer, Mind the Gap!, Schriften der FGNM, 2011, S. 308.

17 So auch Döhl, Ästhetische und juristische Grauzone. Zum Mashup in der Musik am Beispiel des Grey Albums in: Mundhenke/Ramos Arenas/Wilke (Hrsg.), Mashups: Neue Praktiken und Ästhetiken in populären Medienkulturen, 2015, S. 138 mit Blick auf Mashups.

18 Claussen, Remixing YouTube in: Djordjevic/Dobusch (Hrsg.), Generation Remix. Zwischen Popkultur und Kunst, 2014, S. 81; Peifer, Appropriation Art und Fan Art - geknebelte Kreativität oder klare Urheberrechtsverletzung? in: FS Wandtke, 2013, S. 101; Klass, ZUM 2016, 801; Peifer, ZUM 2016, 805; Podszun, ZUM 2016, 606; Reißmann/Klass/Hoffmann, POP Kultur und Kritik 2017, S. 156-172; Klass, Die Referenz als Teil der Kunstform: populärkulturelle Praktiken und das Urheberrecht in: FS Gernot Schulze, 2017, S. 147. 
einander: das Eigentumsrecht (Art. 14 GG) und das (Urheber-)Persönlichkeitsrecht (Art. 2 Abs. 1 i.V.m. Art. 1 Abs. 1 GG) des Schöpfers, der die Kontrolle über sein Werk nicht verlieren sowie keine Ausschließlichkeitsrechte preisgeben will, auf der einen Seite, sowie das Recht auf Zugang und auf Auseinandersetzung mit vorbestehenden Werken derjenigen, die die Referenz als Teil ihrer schöpferischen Freiheit begreifen, auf der anderen Seite. Auch ihre Interessen erfahren i.d.R. durch die Kunstfreiheit (Art. 5 Abs. 3 GG) und durch die Meinungsfreiheit (Art. 5 Abs. 1 GG) verfassungsrechtlichen Schutz. ${ }^{19}$

Im Vordergrund der Auseinandersetzung steht mithin die Frage, wie viel Freiheit das Urheberrecht und die Leistungsschutzrechte den transformativen, derivativen Nutzungen fremder Werke gewähren bzw. gewähren müssen, und welche Grenzen das Recht den Nachschaffenden setzt.

Mit dieser schwierigen Frage musste sich im Jahr 2016 auch das Bundesverfassungsgericht und jüngst der $\mathrm{EuGH}^{20}$ in dem viel beachteten Sampling-Verfahren Metall auf Metall befassen. ${ }^{21}$ Hintergrund dieses, die Gerichte seit mehr als 20 Jahren beschäftigenden Streits ist die Übernahme einer zwei-sekündigen Rhythmussequenz aus der Tonspur des Musikstücks „Metall auf Metall“ der Band „Kraftwerk“ in das Hip-Hop-Stück „Nur mir““.22

Kernpunkt des Streits war die Frage, ob die Kunstschaffenden in fremde Ausschließlichkeitsrechte und damit in Eigentumspositionen eingreifen, und wenn ja, ob dies vor dem Hintergrund der Kunstfreiheitsgarantie zu rechtfertigen ist. ${ }^{23}$

Die Frage, wie ein Ausgleich zwischen den berechtigten Interessen der Rechteinhaber vorbestehender Werke und der verwendenden Künstler herzustellen ist, ist allerdings - dies zeigen beispielsweise die Entscheidungen Germania $3^{24}$ und Goldrapper ${ }^{25}$ - keine neue Frage. Auch handelt es sich nicht um ein deutsches Phänomen, vielmehr zeigt sich der

19 Siehe hierzu auch Schulze, Gedanken zur freien Benutzung und zu einer allgemeinen Grundrechtsschranke am Beispiel Metall auf Metall in: FS Walter, 2018, S. 504 sowie BVerfG ZUM 2016, 626 - Metall auf Metall; BVerfG ZUM 2000, 867 - Germania 3; BVerfG GRUR 1971, 461 - Mephisto; BVerfG ZUM 2007, 829 - Esra; vgl. des Weiteren Klass, ZUM 2016, 802; Podszun, ZUM 2016, 606.

20 EuGH v. 29. Juli 2019, C-476/17 - Metall auf Metall.

21 BVerfG GRUR 2016, 690 - Metall auf Metall.

22 Der Instanzengang: LG Hamburg, Urt. v. 8.10.2004 - 308 O 90/99; OLG Hamburg, Urt. v. 7.6.2006 - 5 U 48/05 = ZUM 2006, 758; BGH, Urt. v. 20.11.2008 - I ZR 112/06 = ZUM 2009, 219; OLG Hamburg, Urt. v. 17. 17.08. 2011 - 5 U 48/05 = ZUM 2011, 748; BGH, Urt. v. 13.12.2012 - ZR 182/11 = ZUM 2013, 484; BVerfG, Urt. v. 31.5.2016 - I BvR 1185/13 = ZUM 2016, 626; BGH, Urt. v. 1.06.2017, I ZR 115/16 = ZUM 2017, 760. Das Verfahren wurde ausgesetzt und dem EuGH vorgelegt, siehe Schlussanträge des Generalanwalts Szpunar vom 12.12.2018, EuGH - C-476/17 - Metall auf Metall, nach Vorlage des: BGH GRUR 2017, 895 Metall auf Metall III sowie aktuell EuGH v. 29. Juli 2019, C-476/17 - Metall auf Metall.

23 Siehe hierzu ausführlich: BVerfG ZUM 2016, 626 - Metall auf Metall sowie Schulze, Gedanken zur freien Benutzung und zu einer allgemeinen Grundrechtsschranke am Beispiel Metall auf Metall in: FS Walter, 2018, S. 504.

24 BVerfG ZUM 2000, 867 - Germania 3.

25 BGH GRUR 2015, 1189 - Goldrapper. 
Konflikt zwischen Eigentumspositionen und künstlerischen Interessen auch in anderen Rechtsordnungen.

So musste beispielsweise das US-amerikanische Berufungsgericht für den 9. Gerichtsbezirk über ein 0,23 Sekunden langes Sample, das Madonna in dem Lied „Vogue“ verwendet hatte, entscheiden. ${ }^{26}$ Und auch Led Zeppelin sah sich dem Vorwurf ausgesetzt, die Melodie des legendären Intros von „Stairway to heaven“, einem Song, der nach Schätzungen ca. 525 Millionen Dollar in Royalties eingespielt haben soll, von der Band „Spirit“ übernommen zu haben. ${ }^{27}$

Aber auch im Bereich der Appropriation Art kommt es immer wieder zu Verfahren. Verantworten musste sich beispielsweise der berühmte Pop-Art Künstler Jeff Koons in den Jahren $2017^{28}$ und $2018^{29}$ in Frankreich, weil Skulpturen aus seiner Ausstellung „Banality“ (1988) als Verletzungen der Urheberrechte zweier französischer Fotografen qualifiziert wurden. ${ }^{30}$ Etwas großzügiger urteilte hingegen das Tribunale Milano, das sich mit einer Klage der Erben des Bildhauers Alberto Giacometti gegen den US-amerikanischen Künstler John Baldessari auseinandersetzen musste. ${ }^{31}$ Aber auch in den USA waren die Gerichte gezwungen, die Grenze dieser speziellen und gezielt in Zuordnungspositionen eingreifenden Kunstform festzulegen. Im Fokus der Auseinandersetzungen standen hier insbesondere Werke von Richard Prince. ${ }^{32}$ Und auch der kanadische Gesetzgeber setzte sich mit dem Phänomen des ,user generated content“ auseinander und reagierte mit der Einführung einer Schranke für nutzergenerierte transformative Inhalte auf die aktuellen Schaffensrealitäten. ${ }^{33}$

Festgehalten werden kann daher, dass die veränderten Schaffensgegebenheiten und die Zunahme nutzergenerierter, derivativer Inhalte das Urheberrecht weltweit vor große Herausforderungen stellen und viele Fragen offen sind. Die bestehende Rechtsunsicherheit

26 Court of Appeals for the 9th Circuit, 824 F.3d 871 (2016) = GRUR Int. 2016, $943-V M G$ v. Madonna; hierzu: Kocatepe, GRUR Int. 2018, 11; Sampling wurde in den USA bereits 1991 Gegenstand eines Verfahrens Biz Markie: United States District Court for the Southern District of New York, 780 F. Supp. 182 (S.D.N.Y. 1991) - Grand Upright Music, Ltd v. Warner Bros. Records Inc. Siehe hierzu ausführlich den Beitrag von Apel, S. 62.

27 Pennsylvania Eastern District Court, Case No. 14-3089 (2015) - Skidmore v. Led Zeppelin; United States Court of Appeals for the 9th Circuit, Case No. 16-56057 (2001) - Skidmore v. Led Zeppelin.

28 TGI Paris, Urt. v. 9.3.2017 - Bauret c/ Jeff Koons.

29 TGI Paris, Urt. v. 8.11.2018 - Davidovici c/ Jeff Koons.

30 Siehe zur Rechtslage in Frankreich sowie zu diesen Verfahren den Beitrag von Lucas-Schloetter, S. 99.

31 Trib. Milano, Urt. v. 13.7.2011 - Giacometti/ Baldessari.

32 Court of Appeals for the 2nd Circuit, 714 F. 3d 694 (2013)= GRUR Int. 2013, 1172 ff. - Cariou v. Prince. Richard Prince transformierte Werke des bekannten Fotografen Patrick Carious aus der Sammlung „Yes Rasta“; Southern District Court of New York, Case No. 15-CV-10160 (2017) Graham v. Prince. In diesem Fall stellte der Künstler in der Gagosian Gallery in New York kommentierte Screenshots von Instagram Profilen anderer Nutzer aus, unter denen sich auch die Fotografie „Rastafarian smoking a joint“ von Donald Graham befand.

33 Sec. 29.21 Copyright Act of Canada (auch bekannt als „YouTube-Schranke“); hierzu: Kocatepe, GRUR Int. 2017, 400. Siehe hierzu ausführlich den Beitrag von Kocatepe, S. 86. 
kann jedoch nicht nur das Werkschaffen professioneller Künstler, sondern auch die kreative Auseinandersetzung von Laien erheblich hemmen und so den öffentlichen Diskurs nachteilig beeinflussen. Im Einzelfall kann es sogar dazu kommen, dass die Authentizität eines Werkes leidet oder auf das Erschaffen des Werkes verzichtet wird, weil eine Rechteklärung unmöglich und die verbleibende Rechtsunsicherheit zu hoch ist. Hinzu kommt, dass sich digitale Praktiken der Bezugnahme primär im Internet abspielen, sodass selten nur eine Rechtsordnung betroffen ist. ${ }^{34}$ Nicht zuletzt wird die rechtssichere Beurteilung der Problematik auch dadurch erheblich erschwert, dass ästhetische Fragen oftmals nur anhand des konkreten Einzelfalls zu beurteilen sind. ${ }^{35}$

Dass eine Klärung der offenen Fragen in einer digitalen vernetzten Welt eine hohe Relevanz hat, zeigten nicht zuletzt auch die heftigen Reaktionen ${ }^{36}$ auf die umstrittene EU-Urheberrechtsreform. ${ }^{37}$

Eine global geführte Debatte über die Möglichkeiten und Grenzen der Nutzung vorbestehender Werke im Rahmen künstlerischer Auseinandersetzungen ist daher mit Blick auf die für alle Akteure bestehende Unsicherheit unerlässlich. ${ }^{38}$

34 Hierzu: Maier, Remixe auf Hosting-Plattformen, 2018, S. $101 \mathrm{ff}$.

35 Je nachdem, wie weit die Bezugnahme geht, ist eine Zustimmung des Originalurhebers notwendig. Die Grenze zwischen der zustimmungsfreien ,freien Benutzung“ nach § 24 UrhG und der zustimmungspflichtigen „Bearbeitung“ nach $\S 23$ UrhG ist nach bisheriger Ansicht fließend; vgl. Schulze in: Dreier/Schulze (Hrsg.), UrhG, 2018, § 24 UrhG Rn. 1. Zur Frage der Vereinbarkeit des § 24 UrhG mit dem Europarecht siehe aktuell EuGH v. 29. Juli 2019, C-476/17 - Metall auf Metall.

36 Siehe als repräsentative Beispiele für die Debatte die Positionen der Kampagne SaveYourInternet: https://saveyourinternet.eu/ (zuletzt abgerufen am 13.3.2019) sowie der Initiative Urheberrecht: https://urheber.info/aktuelles/2019-02-26_initiative-urheberrecht-ein-guter-tag-fuer-europa (zuletzt abgerufen am 13.3.2019). Siehe hierzu aber auch: Suwelack, MMR 2018, 582; Kastl, GRUR 2016, 671; Hofmann, GRUR 2018, 21; Drexl, ZUM 2017, 529.

37 Richtlinie (EU) 2019/790 des Europäischen Parlaments und des Rates vom 17. April 2019 über das Urheberrecht und die verwandten Schutzrechte im digitalen Binnenmarkt und zur Änderung der Richtlinien 96/9/EG und 21/29/EG, siehe: https://eur-lex.europa.eu/legal-content/DE/TXT/ PDF/?uri=CELEX:32019L0790\&from=EN sowie die Analyse und Darstellung der umstrittenen Punkte unter https://emr-sb.de/ aktuelles-stichwort-zur-eu-urheberrechtsreform-und-artikel-13/.

38 Vgl. Dreier in: Dreier/Schulze (Hrsg.), UrhG, 2018, Einl. Rn. 22. 